\title{
EXAMINATION OF GUTTA-PERCHA CONES FOR MICROBIAL CONTAMINATION DURING CHEMICAL USE
}

\author{
Guven KAYAOGLU ${ }^{1}$, Mügem GÜREL ${ }^{1}$, Hüma ÖMÜRL Üํํㄹ Zeliha Gonca BEK ${ }^{1}$, Burak SADIK ${ }^{1}$
}

1- Department of Endodontics and Conservative Treatment, Faculty of Dentistry, Gazi University, Ankara, Turkey.

Corresponding address: Guven Kayaoglu, Department of Endodontics and Consevative Treatment, Faculty of Dentistry, Gazi University, 82. sokaki, 06510, Emek, Ankara, Turkey - e-mail: guvenk@gazi.edu.tr

Received: May 8, 2008 - Modification: August 31, 2008 - Accepted: October 7, 2008

\begin{abstract}
$O$

bjective: The aim of this study was to evaluate the degree of microbial contamination in packaged gutta-percha cones before and during use in clinical conditions. Material and Methods: Sealed packages of \#15-40 gutta-percha cones were opened under aseptic laboratory conditions. Two gutta-percha cones from each size were randomly drawn and added to tubes containing glass beads and $750 \mu \mathrm{L}$ of saline. The tubes were vortexed, serially diluted and samples of $250 \mu \mathrm{L}$ were cultured on agar plates. The plates were incubated at $37^{\circ} \mathrm{C}$ for 3 days and colonies were counted. The initially sampled packages were distributed to 12 final year dental students. The packages were collected at the end of the first and the third clinical practice days and sampled as described above. Results: Baseline microbial counts did not exceed $3 \mathrm{CFU}$. At the end of the first and the third day, additional contamination was found in five and three of the packages, respectively. The ratio of contaminated packages at the first day and the third day was not significantly different ( $z$-test; $p>0.05)$. The numbers of microorganisms cultured at the first day $(8 \pm 9.9 \mathrm{CFU})$ and the third day $(4.5$ $\pm 8.3 \mathrm{CFU}$ ) were not significantly different (Wilcoxon signed-rank test; $p>0.05$ ). No significant correlation was found between the number of filled root canals and cultured microorganisms at either the first day (Spearman's rho; $r=0.481, p=0.113$ ) or the third day $(r=-0.034, p=0.917)$. Conclusions: Gutta-percha cones taken directly from manufacturer's sealed package harbored microorganisms. Clinical use of the packages has been found to be associated with additional contamination of the gutta-percha cones. The counts of cultured microorganisms did not correlate well with the number of filled root canals.
\end{abstract}

Key words: Bacteria. Contaminant. Disinfection. Sterility.

\section{INTRODUCTION}

The purposes of endodontic treatment are to remove all necrotic and vital tissue and microorganisms from the root canal system and obturate the canal space tightly to avoid microbial growth ${ }^{13}$. In this context, the filling materials should also be free of microorganisms to avoid canal recontamination.

Currently, gutta-percha is the most commonly used root canal core filling material. The sap of the isonandra gutta tree is the raw material for gutta-percha. Chemically, it is defined as the trans-form of polyisoprene and is harder, more brittle and less elastic than the more familiar natural rubber. The familiar consistency of endodontic gutta-percha cone is achieved by the addition of wax and resins as softeners (1-4\%), metal sulfate for radiopacity (1-15\%), and zinc oxide filler as the main component $(59-76 \%)^{2}$. Gutta-percha cone possesses some antimicrobial activity owing to its zinc oxide component ${ }^{8}$. In spite of this antimicrobial activity, two studies have reported that $8 \%$ and $20 \%$ of gutta-percha cones taken out of their sealed package yielded bacterial growth when cultured on agar plates, respectively ${ }^{7,10}$. Contrary to these findings, others have not detected microbial contamination on gutta-percha cones taken from the manufacturer's sealed package ${ }^{3,6,12}$. However, it is expected that contamination would increase upon opening of the package and starting to use the gutta-percha cones in the clinical environment. While some studies have investigated the microbial contamination of gutta-percha packages that have been opened and used in a clinic for a certain period ${ }^{4,12}$, the present study, differently, recorded the number of root canals filled as an indicator to determine the frequency of exposure to the potential contaminants. This study examined the degree of microbial contamination on gutta-percha cones at the time of package opening and during clinical use, and also verified whether a correlation existed between the number of filled root canals and the number of the contaminating microorganisms.

\section{MATERIAL AND METHODS}

Initial Sampling and Culturing

Twelve sealed packages of \#15-40 gutta-percha cones 
(Sure-endo, Sure-dent Co., Seoul, Korea) were opened under aseptic laboratory conditions and two gutta-percha cones from each size was randomly drawn. Twelve cones chosen in this manner from each package (two of each \#15, \#20, $\# 25$, \#30, \#35 and \#40 cones) were added to tubes containing $750 \mu \mathrm{L}$ of sterile $0.9 \%$ saline and glass beads (Figure 1). The tubes were vortexed for $30 \mathrm{~s}, 10$-times serially diluted to $10^{-1}$, and samples of $250 \mu \mathrm{L}$ were cultured on tryptone soy agar plates (TSA; Oxoid, Basingstoke, UK). Plating was done on two separate agar plates. The plates were incubated at $37^{\circ} \mathrm{C}$ for 3 days and microbial colonies were counted under stereomicroscope at 6x magnification (Olympus SZPT, Tokyo, Japan). Average of two plates was recorded. As a negative control group, tubes containing only glass beads and saline were sampled. As a positive control group, guttapercha cones rolled between ungloved fingers for $30 \mathrm{~s}$ were sampled as described above.

In a separate test, the whole gutta-percha contents of three sealed packages were sampled. However, in this test, the gutta-percha cones removed from the packages were added to tubes containing $1.5 \mathrm{~mL}$ saline, and volumes of $500 \mu \mathrm{L}$ were pipetted and cultured. Microbial detection limit of the study was 3 colony forming units (cfu).

\section{Participants and the Experimental Design}

The initially sampled packages were distributed randomly to 12 final year dental students (one package per student). The students were anonymous to the author who carried out the laboratory tests. The students were blinded to the aim of the study. They were advised to use only the given package and work as usual. The students were also asked to record the number of root canals they filled with the given gutta-percha packages. The packages were collected at the end of the first and the third day and sampled as described above. At each laboratory setting, three negative and one positive control were run.

\section{Statistical Analysis}

Differences between the number of root canals filled and also between the number of microorganisms cultured at the end of the first and third days were analyzed statistically by the Wilcoxon signed-rank test. The correlation between the number of microorganisms cultured and the number of filled root canals was analyzed using the Spearman's rank correlation coefficient (Spearman's rho). Differences between the ratio of the contaminated packages at the first and third days were analyzed using the $z$-test. The statistical significance was set to 0.05 .

\section{RESULTS}

No colony formation was found for the negative controls $(n=9)$. The positive controls showed microbial growth $(84$, 120 and $135 \mathrm{cfu} ; \mathrm{n}=3)$. The samples of the whole content of the packages displayed microbial growth (12, 15 and 18 $\mathrm{cfu} ; \mathrm{n}=3$ ), confirming that the package contents were not sterile.

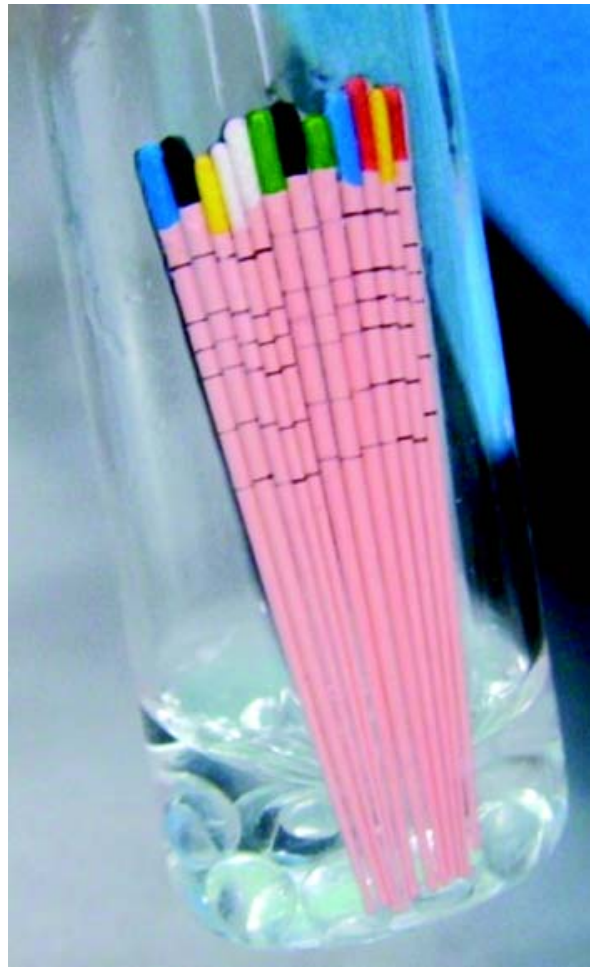

FIGURE 1- Gutta-percha cones (\#15-40) sampled in a tube in the presence of glass beads and saline

Initial samples of the packaged gutta-percha cones showed either no microbial growth ( 7 of 12 packages) or as few as 3 cfu of microbial growth (5 of 12 packages; Table 1). Because the baseline cultures could yield up to $3 \mathrm{cfu}$, this number was considered as the threshold, and further cultures not exceeding $3 \mathrm{cfu}$ were considered to have got no extra contamination.

Descriptive statistics for the first and third day numbers of root canal fillings and cultured microorganisms are shown in Table 2. The number of root canals filled at the end of the first day and the third day were $3.9 \pm 1.1$ and $11.3 \pm 2.9$ (mean $\pm \mathrm{SD}$ ), respectively. Difference between the number of filled root canals at the first and the third day was significant (Wilcoxon test; $p=0.001$ ). The number of microorganisms recovered at the first and third day cultures were $8 \pm 9.9 \mathrm{cfu}$ and $4.5 \pm 8.3 \mathrm{cfu}$ (mean $\pm \mathrm{SD}$ ), respectively. Differences between the numbers of microorganisms found at the first and third day cultures were not significant (Wilcoxon test; $p=0.304$ ). No significant correlation was found between the number of filled root canals and cultured microorganisms at either the first day (correlation coefficient, significance; $\mathrm{r}=0.481, \mathrm{p}=0.113)$ and the third day $(\mathrm{r}=$ $0.034, \mathrm{p}=0.917)$.

At the end of the first day, microbial contamination was found in five packages (42\%). At the end of the third day, microbial contamination was found in three packages $(25$ $\%$ ). Difference between the ratio of the contaminated packages at the first day and the third day was not statistically significant $(z$-test; $p=0.386)$. No contamination was found throughout the study for five of the packages. The microbial status of the packages used by the students and the number 
TABLE 1- Microbial counts at 0, 1-day and 3-day clinical use of the gutta-percha packages distributed to 12 students coded from $A$ to $L$. The values in parentheses indicate the number of root canals filled at the end of the day. The values in parentheses for the third day row indicate the sum of root canals filled from day 1 to day 3 .

\begin{tabular}{lcccccc}
\hline & A & B & C & D & E & F \\
\hline Baseline & 0 & 3 & 3 & 3 & 0 & 0 \\
$1^{\text {st }}$ day & 0 & 3 & 3 & 12 & 27 & 24 \\
& $(3)$ & $(3)$ & $(3)$ & $(4)$ & $(5)$ & $(4)$ \\
$3^{\text {rd }}$ day & 3 & 0 & 6 & 0 & 3 & 0 \\
& $(9)$ & $(13)$ & $(10)$ & $(9)$ & $(7)$ & $(12)$ \\
\hline \multirow{2}{*}{ Baseline } & $\mathbf{G}$ & $\mathbf{H}$ & $\mathbf{I}$ & $\mathbf{J}$ & $\mathbf{K}$ & $\mathbf{L}$ \\
$1^{\text {st }}$ day & 0 & 0 & 0 & 3 & 3 & 0 \\
& 0 & 18 & 3 & 6 & 0 & 0 \\
$3^{\text {rd }}$ day & $(3)$ & $(5)$ & $(3)$ & $(5)$ & $(6)$ & $(3)$ \\
& 0 & 6 & 3 & 0 & 3 & 30 \\
& $(8)$ & $(13)$ & $(13)$ & $(17)$ & $(14)$ & $(10)$ \\
\hline
\end{tabular}

TABLE 2- Descriptive statistics for the first and the third day variables (cfu: colony forming units of microorganisms, and root canal fillings) and statistical significance of the differences between these values

\begin{tabular}{lllccccc}
\hline & & Min & Max & Median & Mean & SD & Statistical significance \\
\hline \multirow{2}{*}{ cfu } & $1^{\text {st }}$ day & 0 & 27 & 3 & 8 & 9.9 & $\mathrm{p}>0.05$ \\
\multirow{2}{*}{ Fillings } & $3^{\text {rd }}$ day & 0 & 30 & 3 & 4.5 & 8.3 & \multirow{2}{*}{$\mathrm{P}<0.05$} \\
& $1^{\text {st }}$ day & 3 & 6 & 3.5 & 3.9 & 1.1 & 2.9 \\
\hline
\end{tabular}

of root canals filled using these packages have been shown in Table 1.

\section{DISCUSSION}

The major findings of this study were that the contents of the gutta-percha packages were not sterile and that further microbial contamination could occur upon clinical use of the packages. While the number of microorganisms was quite small at the beginning, clinical use of the gutta-percha packages led to increased microbial contamination. However, no significant correlation existed between the number of filled root canals and the number of microorganisms cultured.

The experiment exhibited some confounding findings. For example, contamination was found in four packages (packages D, E, F and J; Table 1) in the first day cultures. However, the third day cultures of the same packages revealed no contamination despite the larger number of filled root canals. Similarly, the third day culture of one package (the $\mathrm{H}$ package) yielded fewer microbial colonies than the first day culture of the same package. One explanation may be that the microbial contamination did not affect the whole content of the package evenly; it is possible that while some of the gutta-percha cones in the package became contaminated during clinical use, other cones remained free of contamination.

The findings of this study on the initial microbial status of the gutta-percha cones are in agreement with those of Montgomery $^{7}$ and Namazikhah, et al. ${ }^{10}$, who found that some of the gutta-percha cones taken from the manufacturer's sealed package harbored microorganism, but differ from those of Doolittle, et al. ${ }^{3}$, Klager and Dupont ${ }^{6}$ and Pang, et al. ${ }^{12}$, who found that none of the gutta-percha cones taken from the manufacturer's sealed package were positive for microbial growth. There are, however, methodological differences among these studies. In addition, the brands of the gutta-percha packages tested in these experiments are different. There may be differences in the manufacturing technology among the manufacturers in terms of aseptic production and packaging. The latter statement is supported by Gomes, et al. ${ }^{4}$, who found that the contents of freshly opened gutta-percha packages of one brand could be negative while the contents of another brand could be positive for microbial growth. 
It was expected that the gutta-percha cones would become contaminated upon opening of the packages and starting their clinical use. As expected, the contamination occurred in seven of the twelve packages within a total of three days. In a previous study ${ }^{12}, 29$ of 150 gutta-percha cones $(19.4 \%)$ that had been in clinical use for three months prior to the microbiological sampling were found to be positive for microbial growth. This finding is consistent with presented study, confirming that the clinical use of the guttapercha package is associated with the risk of contamination. However, five of the packages (packages A, B, G, I and K; see Table 1) remained at or below the threshold limit $(3 \mathrm{cfu})$ throughout the study. The finding that packages used by certain students remained non-contaminated throughout the study may be explained by the awareness of these students of the importance of maintaining aseptic conditions during treatment. The possible limitation of the sampling method certainly cannot be excluded.

Unexpectedly, no strong correlation was found between the number of the root canals filled and the number of the cultured microorganisms. This may also be due to the limitation of the sampling method. Additionally, it may be speculated that the antimicrobial effects of gutta-percha cones $^{1,7}$ could have suppressed the activity of the contaminating microorganisms and kept their cultivable numbers under a certain level. Thus, the number of the cultured bacteria did not parallel the number of the root canal fillings. This lack of correlation has also been reported in a previous study where the number of contaminated guttapercha cones remained almost constant within up to 2.5 years of clinical use ${ }^{4}$.

Current root canal obturation techniques involve the use of a sealer along with gutta-percha. Root canal sealers have considerable antimicrobial activity particularly in direct contact with bacteria ${ }^{5}$. Thus, microorganisms contaminating the gutta-percha cones can be eliminated after coating the cones with the sealer. In a previous study ${ }^{10}$, no microorganism was cultured from gutta-percha cones coated with an epoxy resin-based sealer whereas some of the noncoated cones yielded microbial growth. In addition to the antimicrobial effect of the sealer, several chemicals such as $70 \%$ alcohol, sodium hypochlorite, glutaraldehyde, benzalkonium chloride, paraformaldehyde, formocresol, polyvinylpyrrolidone-iodine have been proposed for guttapercha disinfection ${ }^{6}$. Among these chemicals, sodium hypochlorite, with its rapid antimicrobial action, is a safe and effective medication ${ }^{4,9,11,12}$.

The findings of the present study are in line with those of previous studies that have addressed the risk of microbial contamination on gutta-percha cones and sought solutions to overcome this problem ${ }^{3,4,6,7,9-12}$. Our results indicate the need for maintaining careful handling of the gutta-percha packages: the package should be kept closed when not used and a sterile instrument should be used for taking out guttapercha cones from the package. If contamination is suspected, chemical disinfection should be considered.

\section{CONCLUSION}

It is concluded that gutta-percha cones taken directly from the manufacturer's sealed package harbored cultivable microorganisms. While the numbers of these microorganisms were quite low at the time of opening of the package, clinical use of the packages increased the number of microorganisms contaminating the gutta-percha cones.

\section{REFERENCES}

1- Attin T, Zirkel C, Pelz K. Antibacterial properties of electron beamsterilized gutta-percha cones. J Endod. 2001;27:172-4.

2- Beer R, Baumann MA, Kim S. Instruments and materials. In: Rateitschak KH, Wolf HF, editors. Color atlas of dental medicine: Endodontology. New York: Thieme; 2000. p. 59-76.

3- Doolittle TP, Rubel RL, Fried I. The effectiveness of common office disinfection procedures for gutta-percha and silver points. N Y State Dent J. 1975;41:409-14.

4- Gomes BP, Vianna ME, Matsumoto CU, Rossi Vde P, Zaia AA, Ferraz $\mathrm{CC}$, et al. Disinfection of gutta-percha cones with chlorhexidine and sodium hypochlorite. Oral Surg Oral Med Oral Pathol Oral Radiol Endod. 2005;100:512-7.

5- Kayaoglu G, Erten H, Alaçam T, Ørstavik D. Short-term antibacterial activity of root canal sealers towards Enterococcus faecalis. Int Endod J. 2005;38:483-8.

6- Klager P, Dupont AA. The significance of environmental contamination of sealer and gutta-percha before endodontic obturation. Oral Surg Oral Med Oral Pathol. 1987;63:606-9.

7- Montgomery S. Chemical decontamination of gutta-percha cones with polyvinylpyrrolidone-iodine. Oral Surg Oral Med Oral Pathol. 1971;31:258-66.

8- Moorer WR, Genet JM. Antibacterial activity of gutta-percha cones attributed to the zinc oxide component. Oral Surg Oral Med Oral Pathol. 1982;53:508-17.

9- da Motta PG, de Figueiredo CB, Maltos SM, Nicoli JR, Ribeiro Sobrinho AP, Maltos KL, et al. Efficacy of chemical sterilization and storage conditions of gutta-percha cones. Int Endod J. 2001;34:435-9.

10- Namazikhah MS, Sullivan DM, Trnavsky GL. Gutta-percha: a look at the need for sterilization. J Calif Dent Assoc. 2000;28:427-32.

11- Ozalp N, Okte Z, Ozcelik B. The rapid sterilization of gutta-percha cones with sodium hypochlorite and glutaraldehyde. J Endod. 2006;32:1202-4.

12- Pang NS, Jung IY, Bae KS, Baek SH, Lee WC, Kum KY. Effects of short-term chemical disinfection of gutta-percha cones: identification of affected microbes and alterations in surface texture and physical properties. J Endod. 2007;33:594-8.

13- Spångberg LSW. Endodontic treatment of teeth without apical periodontitis. In: Ørstavik D, Pitt Ford TR, editors. Essential Endodontology. Wiltshire: Blackwell Science; 2001. p. 211-36. 